\title{
The Role of Small Telescopes in the Discovery and Follow-up of Near Earth Objects
}

\author{
Andrea Boattini \\ Osservatorio Astronomico di Roma, Sede di Monte Porzio Catone, \\ 00040 Monte Porzio Catone (RM), Italy
}

\begin{abstract}
The discovered population of Near Earth Objects (asteroids and comets) consists of more than 1,300 bodies. Small telescopes provided with a large field of view have played a major role in the field, contributing to the discovery of about $95 \%$ of the total known population. We present a brief summary of the steps that have led to the present knowledge and suggestions to improve it. A proposal for accessing archival images to search for previous apparitions is presented.
\end{abstract}

\section{Overview of Near-Earth Objects}

One field of astronomical research where small telescopes have played a fundamental role is the discovery and follow-up of Near Earth Objects (NEOs).

NEOs are conventionally defined as those minor planets, asteroids and comets, whose perihelia $q \leq 1.3 \mathrm{AU}$. NEOs have been divided into four categories, based on their osculating orbital elements (Shoemaker et al., 1979; Tedesco et al. 2000):

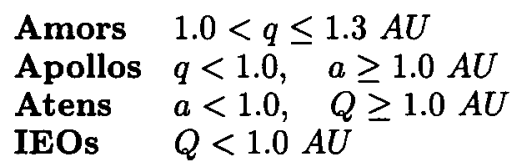

where $a$ is the object's semi-major axis and $Q$ its aphelion distance to the sun; IEOs stands for Interior to the Earth Objects. The cometary component, based on our current knowledge, is much less numerous and will not be addressed in this paper. Comets are conventionally divided into two groups, short and long-period. As of February 28, 2001, the asteroidal component of NEOs, Near Earth Asteroids (or NEAs), include almost 1,300 bodies, with sizes ranging from $30-\mathrm{km}$ to less than 10 meters (The NEO Page).

The most recent studies estimate that there are about 1,000 NEAs of $1 \mathrm{~km}$ or bigger and a much higher number at smaller sizes. Only $40 \%$ of the $\mathrm{km}$-sized and a minor fraction of the smaller objects are known; furthermore good orbits are not available for all of them.

\subsection{What is Needed for the Study of NEOs}

Our knowledge of NEOs has been achieved through three major steps: discovery, follow-up and space studies. They can be briefly outlined according to the fol- 
lowing scheme: a) Discovery - photographic (sporadic, systematic from 1973 to 1996), lim. mag. $15-18.5 \mathrm{~V}$ or $C C D$ (from 1989, large field of view (FOV) from 1996), lim. mag. 18 - 22 V; b) Follow-up - astrometric - optical (phot., CCD), radar; physical - morphological, dynamical and miralogical characterization; $d y$ namical - long and short-term evolution, collision analysis; c) Space missions - physical studies - radiometry, thermal infrared studies, near-IR spectroscopy, spectrophotometry; threat mitigation and discovery.

\section{The Role of Small Telescopes in the Discovery of NEOs}

Astrometric and photometric work is where small telescopes have played a leading role. NEO discovery is not an easy task to conduct successfully: because of their vicinity to the Earth during their visible apparitions, these objects can appear anywhere in the celestial sphere. They also tend to move pretty fast across the sky and remain visible for only a short period of time. In order to conduct a successful NEO search program the two most important requirements are: i) to sweep as much of the sky as possible in a limited amount of time and to inspect the data in near real time; ii) to reach relatively faint magnitudes with short exposures, since the limiting magnitude of NEOs is set by their apparent angular speed in the sky. For this reason Schmidt telescopes and astrographs have been among the most successful instruments when photography has been used, while other optical configurations, such as short focal length Cassegrain and Newton systems have proven their potential with CCD systems. NEOs have been discovered during the course of programs with different objectives (Helin and Dunbar 1990). We can determine three distinct historical periods:

1900-1970: incidental discoveries, made in the course of programs initiated for other objectives, such as mapping the sky in different colours, or studying the proper motions of stars. Most of these discoveries were achieved in the course of general asteroid surveys, not directly addressed to NEOs.

1970-1990: first NEO search programs. In the early 1970 s the first dedicated NEO search program was started at Mount Palomar using the 0.46-m Schmidt. Two programs operated this telescope, PCAS and PACS and contributed to about $50 \%$ of all the photographic discoveries. The Australian project AANEAS was also quite successful (Steel et al. 1997). In 1983 two NEAs were discovered by the infrared satellite IRAS. The most interesting result was to obtain a first inventory and characterization of the NEO population, its size distribution and a hazard assessment.

1990-2001: second period of NEO search programs. The transition between the first and the second period started about ten years ago, when the scientific community realized that in order to discover the great majority of $\mathrm{km}$ sized NEOs, the ones that have the potential to cause a global catastrophe, it would have taken more than a century to accomplish this task with photographic methods. To shorten the time of such a survey, called the Spaceguard Survey (Morrison 1992), to only one or two decades, electronic devices such as large format CCDs or CCD mosaics have to be used because of their digital processing capacity, greater dynamic range and quantum efficiency. After the pioneering work of the Spacewatch program (Scotti 1993), the only CCD program between 

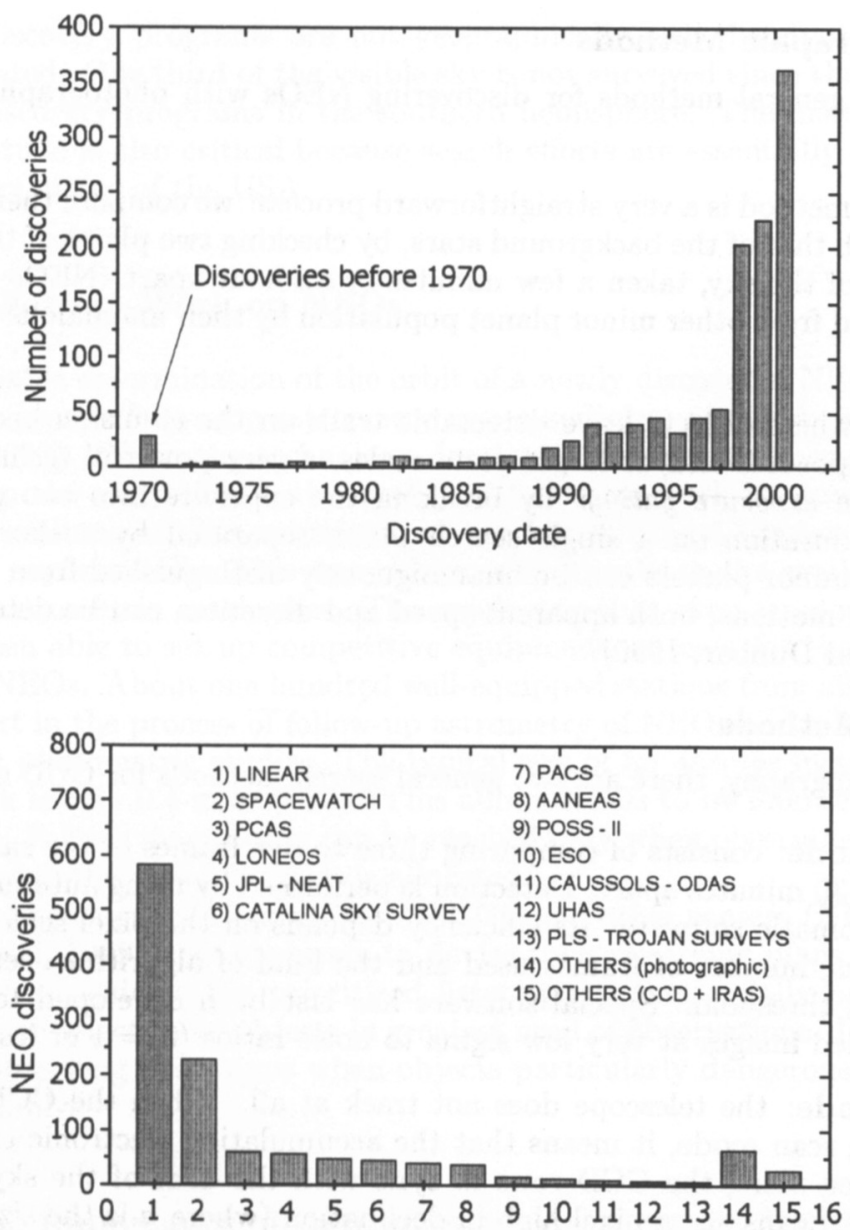

Figure 1. The upper plot shows the number of NEA discoveries per year in the course of the last century. The first NEA, (433) Eros, was discovered in 1898. We can see a slow but constant rise in the discovery rate by the photographic programsin the 1970s and 1980s, before the great jump in $1997 / 1998$, when the wide-field CCD programs started their operations. The lower plot shows the most successful programs as of February 28, 2001. LINEAR has been the most productive so far, and in the past three years has been credited with the discovery of two-thirds of all NEAs. CCD survey programs cover two-thirds of the visible sky to a magnitude of $18-19 \mathrm{~V}$ about twice a month. 
1989 and 1996, further CCD projects were started and have provided very successful results (The NEO Page; JPL-NEOP). Only in 1997 there was the first NEO discovery with a telescope bigger than two meters and only in 2000 the first such discovery was made with a four meter class instrument.

\subsection{Photographic Methods}

There are two general methods for discovering NEOs with photographic techniques:

- The first method is a very straightforward process: we compare their movement with that of the background stars, by checking two plates of the same location of the sky, taken a few minutes or an hour apart. NEOs are distinguished from other minor planet population by their anomalous angular speed.

- In cases where objects leave detectable trails on the emulsion because of longer exposures and/or larger plate scales, a very powerful technique to use is the exposure gating: by breaking the exposure into two parts of unequal duration on a single search plate, separated by a short blank interval, minor planets can be unambiguously distinguished from defects, and their motions, both apparent speed and direction, can be determined (Helin and Dunbar, 1990).

\subsection{CCD Methods}

Similar to photography, there are two general search methods for CCD systems:

- Stare-mode: consists of comparing three to five frames of the same field taken 15-30 minutes apart. Detection is performed by using automatic and semi-automatic software. Its efficiency depends on the pixel scale, seeing conditions, number of frames used and the kind of algorithms set up for detection threshold. Special software has last been developed to detect long trailed images at very low sigma to noise ratios ( $\sigma=1$ or less).

- Scan-mode: the telescope does not track at all. When the CCD is operated in scan mode, it means that the accumulating electronic charge is transferred along the CCD rows in sync with the drift of the sky across the CCD. Scans are $n$ pixel high in declination (where $n$ is the size of the CCD in pixels) and an arbitrary number of rows long in right ascension. A survey region consists of three scans of the same length at the same location of the sky. The detection is made in the same way as described for the stare mode approach.

\section{Pitfalls in the Current Knowledge of NEOs}

There are three major pitfalls in the current astrometric activities on NEOs:

- The discovery activity is still not supported by equivalent follow-up work, both astrometric and physical. One of the reasons for this situations is a need of larger telescopes. 
- The present knowledge of NEOs with small aphelia is very limited (Atens), or non existent $(I E O s)$. The reason is that only in the last few years NEO searching has been extended to smaller solar elongations, but a lot more needs to be done: small telescopes can still contribute in this task.

- Discovery programs are not geographically well distributed and coordinated. One third of the visible sky is not surveyed since there are no NEO discovery programs in the southern hemisphere. The distribution in longitude is also critical because search efforts are essentially confined to the dry states of the USA.

\section{Follow-up Work on NEOs}

The reliable determination of the orbit of a newly discovered NEO is impossible without precise follow-up astrometry over a sufficient arc of observations (Ticha et al. 2000). When the technology developed that allowed a rapid diffusion of CCDs in the early 1990's, the quality of follow-up work improved significantly and the volume of data submitted to the Minor Planet Center (MPC) has increased exponentially. This was also possible thanks to the availability of much better astrometric catalogues. The result was that even amateur astronomers have been able to set up competitive equipment, at least for the follow-up process of NEOs. About one hundred well-equipped stations from all over the world take part in the process of follow-up astrometry of NEOs and a few of them also conduct photometric studies. The typical size of an average instrument used in this task is only $0.4-\mathrm{m}$ or $0.5-\mathrm{m}$. This allows NEOs to be followed up to magnitude 20. Fainter magnitudes can be reached only when observing time at better equipped professional facilities is allocated.

The Spaceguard Central Node (SCN), and other centers (NEODyS; Koehn and Bowell 1999) help observers to devise their observing plans. The SCN, for example, provides a few prioritized lists, updated on a daily basis, where observers can select those objects in greatest need of observations. It also organizes direct observing campaigns when objects particularly dangerous risk to be lost prematurely.

\section{The Importance of Astronomical Archives for Cataloging NEOs}

The remarkable increase in the NEO discovery rate and significant improvements of the methods developed by the teams that study collision analysis and shortterm evolution, has led to the discovery and the recognition of a few NEOs with small collision probabilities. In order to assess this danger, the orbits of these bodies must be known with great accuracy and this only can be achieved by obtaining astrometric positions over a long period of time.

The inspection of archival material where one or more images of NEOs, previously undetected, may be present, offers several advantages: i) very good orbits can be obtained soon after discovery; ii) two km-sized NEAs with small collision probabilities were mitigated using data from photographic precovery images (1997 XF11 and 1999 AN10); iii) a great deal of telescope time, as well 
as money is saved; iv) this can be a day-time activity, unaffected by the vagaries of weather;

Although a few dedicated teams routinely look for pre-discovery images of NEOs in a few high quality archives (DANEOPS, ANEOPP), the great bulk of the estimated two million photographic plates/films from professional collections is not easily accessible. In addition, CCD archives are quickly developing that promise to represent a very potential resource in future decades.

\subsection{A Proposal for International Collaboration}

The recent development of NEO scientific work imposes a higher degree of coordination among all the tasks involved to obtain knowledge of these bodies. For the reasons discussed above, the archival work, both collection and organization, needs to be improved. The SCN is trying to organize a proper interface for NEO work. There is a need for an international center, defined by the IAU, eventually sponsored also by other Institutions and agencies that could organize all this archival material, both photographic and CCD. For NEO work it is essential to meet the following requirements:

- A complete list of CCD surveys and various sky patrols initiated for diverse objectives.

- For each program a list of catalog files reporting the epoch of the exposure, the exposure time, the position, the field of view (FOV), and the limiting magnitude

- Easy retrieval of desiderable data in digital form.

The goal of such an effort would be to complete the inventory of NEOs (NEOs with good orbits!) in much less time and to assess the danger posed by these objects using all the available resources.

\section{Future Programs}

Many new projects all around the world are on the horizon for the near future. Asia is starting several programs, with the BATTeRS project in Japan taking a leading role (Isobe, this meeting). I'm involved in an Italian project, CINEOS, that will survey regions at small solar elongations to look for Atens and IEOs. The UK and other European countries are working on additional projects. Australia should see the first NEO survey in the southern hemisphere later this year, as part of the Catalina Sky Survey. In the USA, significant upgrades are expected on the short term at Mount Palomar and in the course of the Spacewatch program. Small telescopes are going to play a leading role, even from space: in order to address the danger of Atens and IEOs more efficiently and to better characterize the physical properties of all NEOs, many space agencies are undertaking a few dedicated missions that will start operations within a decade.

\section{References}

ANEOPP, http://www.arcetri.astro.it/science/aneopp/ 
DANEOPS, http://earn.dlr.de/daneops/

Helin E.F. and Dunbar S.R. 1990, Vistas in Astronomy, 33, 21-37

Koehn B., and Bowell E. 1999, Asteroid Observing Services, http://asteroid.lowell.edu

Minor Planet Center, http://cfa-www.harvard.edu/iau/mpc.html

Morrison D. (editor) 1992, The Spaceguard Survey, NASA Report

NASA-JPL NEO Program, 2001, http://neo.jpl.nasa.gov

NEODyS, http://newton.dm.unipi.it/neodys

The NEO Page, Minor Planet Center,

http://cfa-www.harvard.edu/iau/NEO/TheNEOPage.html

Scotti J.V., Asteroids, Comet, Meteors, 1993, 17-30

Shoemaker E.M., Williams J.G., Helin E.F., Wolfe R.F. 1979, Asteroids, 253-282

Spaceguard Central Node, http://spaceguard.ias.rm.cnr.it

Steel D.I., McNaught R.H., Garradd, G.J., Asher D.J., Russell K.S. 1997, Austr. J. Astron., 7, 67-77

Tedesco E.F., Muinonen K., Price S.D. 2000, P\&SS, 48, 801-816

Ticha J., Tichy M., Moravec Z. 2000, P\&SS, 48, 787-792 